\title{
ISOMORPHISM THEOREMS FOR OCTONION PLANES OVER LOCAL RINGS
}

BY

ROBERT BIX

\begin{abstract}
It is proved that there is a collineation between two octonion planes over local rings if and only if the underlying octonion algebras are isomorphic as rings. It is shown that every isomorphism between the little or middle projective groups of two octonion planes over local rings is induced by conjugation with a collineation or a correlation of the planes when the local rings contain $\frac{1}{2}$.
\end{abstract}

Octonion planes over local rings were defined and studied in [3]. In this paper we prove two main theorems about such planes. In $\$ 1$ we show that there is a collineation between two such planes if and only if there is a semilinear algebra isomorphism of the underlying octonion algebras. This was proved for octonion algebras over fields by Faulkner [6, p. 20].

Second, we prove that every isomorphism between the little or middle projective groups of two octonion planes over local rings is induced by conjugation with a collineation or a correlation of the planes when 2 is a unit in the local rings. This was proved for octonion division algebras over fields of characteristic $\neq 2$ by Suh [10, p. 338] and Veldkamp [11, p. 287]. The corresponding result for octonion division algebras over fields of characteristic 2 was proved by Faulkner [6, p. 57]. Thus our theorem extends known results to split octonion algebras over fields of characteristic $\neq 2$ and to arbitrary octonion algebras over local rings containing $\frac{1}{2}$. In the case of fields of characteristic $\neq 2$, we further generalize known results by extending our theorem to include isomorphisms between subgroups of the full collineation groups of two planes when each subgroup contains the little projective group. This extension follows directly from the classification of the subgroups of the full collineation group normalized by the little projective group [3, Corollary 7.2].

The results of the last paragraph are proved in $\$ \S 2-6$. In $\$ 2$ we study two kinds of involutions in the little projective group $P S$ : those that fix all the points on a line and a point not connected to the line, and those that fix a four-point. We prove in $\$ 3$ that every involution in the middle projective group $P G$ is of one of these two kinds. We show in $\$ 4$ that involutions of the first kind are distinguished in PS by group-theoretic properties, so they are preserved by isomorphisms of little projective groups. In $\$ 5$ we determine the geometric conditions for two involutions of the

Received by the editors May 15, 1980.

AMS (MOS) subject classifications (1970). Primary 17C40.

Key words and phrases. Octonion plane, exceptional Jordan algebra. 
first kind to commute and for their product to be an involution of the first kind. We apply this criterion in $\$ 6$ to prove the results of the preceding paragraph.

All notation is as in [3]. $\subseteq$ is an octonion algebra over a local ring $(R, m)$ with norm $n(x)$, trace $t(x)$, and involution $x \rightarrow x^{d} . J=H\left(\mathfrak{O}_{3}, \gamma\right)$ is the quadratic Jordan algebra of Hermitian 3-by-3 matrices over $\mathfrak{D}$. If $x, y \in J, N(x)$ is the generic norm of $x, x^{\#}$ is the adjoint of $x$, and $x \times y=(x+y)^{\sharp}-x^{\#}-y^{\sharp} . \Gamma=\Gamma(J)$ is the group of norm semisimilarities of $J, G=G(J)$ is the group of norm similarities, and $S=S(J)$ is the group of norm preserving transformations [3, Definition 1.2]. The octonion plane $P J$ consists of points $x_{*}=R x$ and lines $x^{*}=R x$ for $x \in$ $J-m J, x^{\#}=0$, with relations:

$$
\begin{aligned}
& x_{*} \mid y^{*}, x_{*} \text { "on" } y^{*}, \text { if } V_{x_{y}}=0, \\
& x_{*} \sim y^{*}, x_{*} \text { "connected" to } y^{*}, \text { if } T(x, y) \in m, \\
& x_{*} \sim y_{*}, x_{*} \text { "connected" to } y_{*}, \text { if } x \times y \in m J, \\
& x^{*} \sim y^{*}, x^{*} \text { "connected" to } y^{*}, \text { if } x \times y \in m J .
\end{aligned}
$$

If $x_{*} \nsim y_{*},(x \times y)^{*}$ is the unique line on $x_{*}$ and $y_{*}$; if $x^{*} \nsim y^{*},(x \times y)_{*}$ is the unique point on $x^{*}$ and $y^{*}$ [3, Lemma 2.2]. A three-point is an ordered triple of points $\left(a_{1 *}, a_{2 *}, a_{3 *}\right)$ such that $a_{1 *} \nsim\left(a_{2} \times a_{3}\right)^{*}$, a condition symmetrical in the $a_{i}$. A four-point is an ordered quadruple of points such that any three form a three-point. A collineation of two octonion planes consists of a bijection of their points and a bijection of their lines preserving the relations "on" and "connected to". $\phi \in \Gamma(J)$ induces a collineation $P \phi$ of $P J$ by $P \phi\left(x_{*}\right)=(\phi x)_{*}$ and $P \phi\left(y^{*}\right)=$ $\left(\phi^{*-1} y\right)^{*}$. If $H \subset \Gamma$, let $P H=\{P \phi \mid \phi \in H\} . P \Gamma$ is the full collineation group of $P J$, and $R-m$ is the kernel of the homomorphism $\phi \rightarrow P \phi$ taking $\Gamma$ onto $P \Gamma[3$, Lemma 3.3 and Theorem 8.4]. Let $\mathfrak{D}^{\prime}$ and $J^{\prime}$ be defined analogously over a local ring $\left(R^{\prime}, m^{\prime}\right)$.

1. Isomorphism of octonion planes. In this section we prove that there is a collineation between two octonion planes if and only if their underlying octonion algebras are isomorphic as rings. Since every collineation between two octonion planes is induced by a norm semisimilarity [3, Theorem 8.4], it suffices to prove that there is a norm semisimilarity of $J$ onto $J^{\prime}$ if and only if there is a ring isomorphism of $\mathfrak{D}$ onto $\mathfrak{D}^{\prime}$.

Define a norm semisimilarity $(\phi, \sigma)$ of $\mathscr{D}$ onto $\mathfrak{D}^{\prime}$ to be an additive group isomorphism $\phi: \mathfrak{D} \rightarrow \mathfrak{D}^{\prime}$, a ring isomorphism $\sigma: R \rightarrow R^{\prime}$, and a unit $\rho^{\prime} \in R^{\prime}$ such that $\phi(\alpha x)=\alpha^{\sigma} \phi x$ and $n^{\prime}(\phi x)=\rho^{\prime} n(x)^{\sigma}$ for $\alpha \in R$ and $x \in \mathfrak{O}$. If $\phi$ takes $1 \in \mathfrak{D}$ to $1^{\prime} \in \mathfrak{D}^{\prime}$, setting $x=1$ in the last sentence implies that $n^{\prime}(\phi x)=n(x)^{\sigma}$ for $x \in \mathfrak{D}$.

LEMMA 1.1. There is a ring isomorphism of $D$ onto $\mathfrak{D}^{\prime}$ if and only if there is a norm semisimilarity of $\mathfrak{D}$ onto $\mathfrak{D}^{\prime}$ taking $1 \in \mathfrak{D}$ to $1^{\prime} \in \mathfrak{D}^{\prime}$. In fact, any ring isomorphism $\phi$ of $\mathfrak{D}$ onto $\mathfrak{D}^{\prime}$ induces a norm semisimilarity $(\phi, \sigma)$ taking 1 to $1^{\prime}$ such that $(\phi x)^{d^{\prime}}=$ $\phi\left(x^{d}\right)$ and $t^{\prime}(\phi x)=t(x)^{\sigma}$ for $x \in \mathfrak{D}$.

Proof. Let $\phi$ be a ring isomorphism of $\mathscr{O}$ onto $\mathfrak{D}^{\prime} . \phi(R 1)=R^{\prime} 1^{\prime}[3$, Lemma 1.11]. Since $R^{\prime} \cong R^{\prime} 1^{\prime}$, we can define a ring isomorphism $\sigma$ of $R$ onto $R^{\prime}$ by $r^{\sigma} 1^{\prime}=\phi(r 1)$, so that $\phi$ is $\sigma$-semilinear. Let $\mathcal{S}$ have a free basis $1, x_{1}, \ldots, x_{7}$ over $R$. 
Since $\phi x_{i}$ and $1^{\prime}$ are linearly independent, $t^{\prime}\left(\phi x_{i}\right)$ is determined by the equation

$$
\left(\phi x_{i}\right)^{2}-t^{\prime}\left(\phi x_{i}\right) \phi x_{i}+n^{\prime}\left(\phi x_{i}\right) 1^{\prime}=0 .
$$

Applying $\phi$ to the equation $x_{i}^{2}-t\left(x_{i}\right) x_{i}+n\left(x_{i}\right) 1=0$ shows that $t^{\prime}\left(\phi x_{i}\right)=t\left(x_{i}\right)^{\sigma}$. Since $t^{\prime}(\phi 1)=2^{\prime}=t(1)^{\sigma}, t^{\prime}(\phi x)=t(x)^{\sigma}$ for $x \in \mathcal{D}$. Since $x^{d}=t(x) 1-x, \phi\left(x^{d}\right)=$ $(\phi x)^{d^{\prime}}$. Applying $\phi$ to the equation $x x^{d}=n(x) 1$ yields $n^{\prime}(\phi x)=n(x)^{\sigma}$.

Conversely, let $(\phi, \sigma)$ be a norm semisimilarity of $\mathfrak{D}$ onto $\mathfrak{D}^{\prime}$ such that $\phi 1=1^{\prime}$. Since $n(\phi x)=n(x)^{\sigma}$ for $x \in \mathcal{D}$, the equations $t(x)=n(x, 1)$ and $x^{d}=t(x) 1-x$ imply that $t(\phi x)=t(x)^{\circ}$ and $\phi\left(x^{d}\right)=(\phi x)^{d^{\prime}}$ for $x \in \mathfrak{D}$. $n(x)$ is a nondegenerate quadratic form on $\mathfrak{D}$ [3, Definition 1.6]. Take $a \in \mathfrak{D}$ such that $C_{1}=R 1+R a$ is a free module of rank 2 on which $n$ is nondegenerate. Since $a^{2}-t(a) a+n(a) 1=0$, $\phi$ is a ring isomorphism of $C_{1}$ into $\mathfrak{D}^{\prime}$. By induction, assume we have found a subalgebra $C_{t}$ of $\mathfrak{D}$ such that $C_{t}$ is a free $R$-module of rank $2^{t}$, the restriction of $n$ to $C_{t}$ is nondegenerate, and there is a $\sigma$-semilinear algebra isomorphism $\psi_{t}$ of $C_{t}$ into $\mathfrak{D}^{\prime}$ such that $\psi_{t}\left(x^{d}\right)=\left(\psi_{t} x\right)^{d^{\prime}}$. Applying $\psi_{t}$ to $x x^{d}=n(x) 1$ shows that $n^{\prime}\left(\psi_{t} x\right)=$ $n(x)^{\sigma}$. Then $\phi^{-1} \psi_{t} \in \operatorname{Hom}_{R}\left(C_{t}, \mathfrak{D}\right)$ satisfies $n\left(\phi^{-1} \psi_{t} x\right)=n(x)$ for $x \in C_{t} \cdot \phi^{-1} \psi_{t}$ extends to $\eta \in \operatorname{Hom}_{R}(\mathfrak{D}, \mathfrak{D})$ such that $n(\eta x)=n(x)$ for $x \in \mathfrak{D}$, by Witt's cancellation theorem for nondegenerate quadratic forms over local rings [2, p. 80]. Then $\phi \eta$

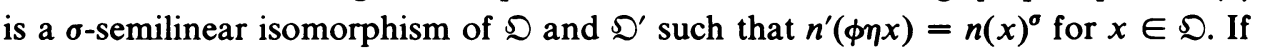
$t<3$, take $p \in C_{t}^{\perp}$ such that $n(p)$ is a unit. $\phi \eta p \in\left(\psi_{t} C_{t}\right)^{\perp}$ and $n^{\prime}(\phi \eta p)=n(p)^{\sigma}$. Set $C_{t+1}=C_{t}+C_{t} p$ and set

$$
\psi_{t+1}(x+y p)=\psi_{t} x+\left(\psi_{t} y\right)(\phi \eta p)
$$

for $x, y \in C_{t}$. The proof of [3, Lemma 1.9] shows that $C_{t+1}$ is a free $R$-module of rank $2^{t+1}, C_{t+1}$ is a subalgebra of $\mathcal{D}, n$ is nondegenerate on $C_{t+1}$, and $\psi_{t+1}$ is a $\sigma$-semilinear ring isomorphism of $C_{t+1}$ into $\mathfrak{S}^{\prime}$ such that $\psi_{t+1}\left(z^{d}\right)=\left(\psi_{t+1} z\right)^{d^{\prime}}$ for $z \in C_{t+1}$, completing the induction. $\psi_{3}$ is a ring isomorphism of $\mathcal{O}$ onto $\mathfrak{D}^{\prime}$, by Nakayama's Lemma [5, p. 7].

If char $R / m \neq 2$, the next lemma follows from [4, Theorem 3.5].

LeMma 1.2. Let $\left\{x_{1}, \ldots, x_{t}\right\}$ span $J$ as an $R$-module and let $R\left[\eta_{1}, \ldots, \eta_{t}\right]$ be $a$ polynomial ring. Let $X=\sum \eta_{i} x_{i} \in J \otimes_{R} R\left[\eta_{i}\right]$. Then $X^{2}, X$, and 1 are linearly independent over $R\left[\eta_{i}\right]$.

Proof. Let $h$ be a positive integer and let $P(h, R)$ be the $R$-submodule of $R\left[\eta_{i}\right]$ composed of polynomials of total degree at most $h$. Let $t_{1}, \ldots, t_{d}$ be the monic monomials of total degree at most $h-2$ in $R\left[\eta_{i}\right]$. The images of the $t_{i} X^{2}, t_{i} X$, and $t_{i} 1$ in

$$
\left(J \otimes_{R} P(h, R)\right) / m\left(J \otimes_{R} P(h, R)\right) \cong(J / m J) \otimes_{R / m} P(h, R / m)
$$

are linearly independent over $R / m\left[6\right.$, p. 10]. Since $J \otimes_{R} P(h, R)$ is a finite free $R$-module, the $t_{i} X^{2}, t_{i} X$, and $t_{i} 1$ are linearly independent over $R$ as elements of $J \otimes_{R} P(h, R)\left[5\right.$, p. 24]. Since $J \otimes_{R} P(h, R)$ is isomorphic to its image in $J \otimes_{R} R\left[\eta_{i}\right]$, the lemma follows. 
THEOREM 1.3. The following conditions are equivalent:

(1) There is a ring isomorphism of $\subseteq$ onto $\mathfrak{D}^{\prime}$.

(2) There is a semilinear algebra isomorphism of an isotope of $J$ onto $J^{\prime}$.

(3) There is a norm semisimilarity of $J$ onto $J^{\prime}$.

Proof. (1) $\Rightarrow(2)$. If $\phi$ is a ring isomorphism of $\mathfrak{D}$ onto $\mathfrak{D}^{\prime}$, the last sentence of Lemma 1.1 implies that there is $\sigma: R \rightarrow R^{\prime}$ such that

$$
\sum \alpha_{i} e_{i}+a_{i}[j k] \rightarrow \sum \alpha_{i}^{\sigma} e_{i}+\phi a_{i} k[j k]
$$

is a $\sigma$-semilinear algebra isomorphism of $H\left(\mathfrak{D}_{3}, 1\right)$ and $H\left(\mathfrak{D}_{3}^{\prime}, 1^{\prime}\right)$. (2) follows, since $Y \rightarrow Y \gamma$ is an isomorphism of the $\gamma$-isotope $H\left(\mathfrak{D}_{3}, 1\right)^{(\gamma)}$ onto $H\left(\mathfrak{D}_{3}, \gamma\right)$. (2) $\Rightarrow$ (3). Let $J^{(u)}$ be the $u$-isotope of $J, u \in J$ invertible, and let $(\phi, \sigma)$ be a semilinear algebra isomorphism of $J^{(u)}$ onto $J^{\prime}$. Let $X \in J^{(u)} \otimes_{R} R\left[\eta_{i}\right]$ be as in Lemma 1.2 and extend $(\phi, \sigma)$ to a semilinear algebra isomorphism of $J^{(u)} \otimes_{R} R\left[\eta_{i}\right]$ onto $J^{\prime} \otimes_{R^{\prime}} R^{\prime}\left[\eta_{i}\right] . X$ satisfies a monic polynomial over $R\left[\eta_{i}\right]$ of degree three with constant term $N(u) N(X)[9$, p. 500]. Applying $\phi$ to this polynomial and taking the corresponding polynomial for $\phi(X)$ shows that $\phi(X)$ satisfies monic polynomials of degree three with constant terms $N(u)^{\sigma} N(X)^{\sigma}$ and $N^{\prime}(\phi X)$. Applying Lemma 1.2 to $\phi X=\sum \phi\left(x_{i}\right) \eta_{i}$ gives $N(u)^{\sigma} N(X)^{\sigma}=N^{\prime}(\phi X)$. Specializing $\eta_{1}=1$ and $\eta_{i}=0$ for $i \geqslant 2$ gives $N(u)^{\sigma} N\left(x_{1}\right)^{\sigma}=N^{\prime}\left(\phi x_{1}\right)$. Since $x_{1}$ is an arbitrary element of $J, \phi$ is a norm semisimilarity. (3) $\Rightarrow(1)$. Let $(\phi, \sigma)$ be a norm semisimilarity of $J$ onto $J^{\prime}$. We can assume that $\phi\left(R e_{i}\right)=R^{\prime} e_{i}^{\prime}$, since $S\left(J^{\prime}\right)$ is transitive on three-points [3, Proposition 2.1]. Then $\phi(\mathfrak{D}[j k])=S^{\prime}[j k]$, by the proof of [3, Lemma 3.2]. Since $N\left(e_{1}+x[23]\right)=-\gamma_{2} \gamma_{3} n(x)$ for $x \in \mathfrak{D}$, define a norm semisimilarity $\psi$ of $\mathfrak{D}$ onto $\mathfrak{D}^{\prime}$ by $(\psi x)[23]=\phi(x[23]) . \psi 1$ is invertible, since $n(\psi 1)$ is a unit. $x \rightarrow(\psi 1)^{-1} \psi x$ is a norm semisimilarity of $\mathfrak{D}$ onto $\mathfrak{D}^{\prime}$ taking 1 to $1^{\prime}$. We are done by Lemma 1.1.

2. Involutions of the first and second kinds. Henceforth we assume that 2 is a unit in $R$. In this section we study two types of involutions (elements of order two) in $P G$. Define an involution of the first kind to be an involution in $P G$ fixing a point $a_{*}$ and all points on a line $b^{*}$, where $a_{*} \nsim b^{*}$. Define an involution of the second kind to be an involution in $P G$ fixing a four-point.

We claim that $P S$ is transitive on pairs $a_{*} \nsim b^{*}$. There are $c_{*}$ and $d_{*}$ on $b^{*}$ such that $c_{*} \nsim d_{*}$, since $P S$ is transitive on lines [3, Proposition 2.1]. $a_{*} \nsim b^{*}=$ $(c \times d)^{*}$, so $\left(a_{*}, c_{*}, d_{*}\right)$ is a three-point. The claim follows, since $P S$ is transitive on three-points [3, Proposition 2.1].

Define $\zeta_{e_{i *}, e_{i}^{*}} \in S$ to be 1 on $R e_{i}+J_{0}\left(e_{i}\right)$ and -1 on $J_{1 / 2}\left(e_{i}\right)$.

Proposition 2.1. If $a_{*} \nsim b^{*}$, there is a unique involution $P \zeta_{a_{*}, b^{*}} \in P G$ fixing $a_{*}$ and all points on $b^{*}$. If $P \phi \in P \Gamma$,

$$
P \phi P \zeta_{a_{*}, b^{*}} P \phi^{-1}=P \zeta_{P \phi a_{*}, P \phi b^{*}}
$$

Involutions of the first kind form a conjugacy class of PS.

Proof. Since $P S$ is transitive on pairs $a_{*} \nsim b^{*}$ and $\zeta_{e_{1 *}, e_{i}^{*}} \in S$, it suffices to prove that $P \zeta_{e_{1 *}, e_{1}^{*}}$ is the unique involution in $P G$ fixing $e_{1 *}$ and all points on $e_{1}^{*}$. Let $P \psi \in P G$ be another such involution. Since $P \psi$ fixes $e_{2 *}$, we can replace $\psi$ by a 
scalar multiple and assume that $\psi$ fixes $e_{2} . P \psi^{2}=1$, so $\psi^{2}$ is scalar multiplication [3, Lemma 3.3]. Then $\psi^{2}=1$, since $\psi$ fixes $e_{2}$. Since $\psi$ fixes each $R e_{i}$, it fixes $\mathscr{D}[23]$ [3, Lemma 3.2]. Because $\psi$ fixes $e_{2}$ and $R\left(e_{2}+a[23]+\gamma_{2} \gamma_{3} n(a) e_{3}\right)$ for all $a \in \mathcal{D}, \psi$ is the identity map on $J_{0}\left(e_{1}\right)$. It follows that $\psi$ is multiplication by $\tau \in R-m$ on $J_{1 / 2}\left(e_{1}\right)$, where $\psi e_{1}=\tau^{2} e_{1}\left[3\right.$, Lemma 5.1]. $\psi^{2}=1$ implies that $\tau^{2}=1 . \tau= \pm 1$, since 2 is a unit in $R$. Since $\psi \neq 1, \tau=-1$ and $\psi=\zeta_{e_{1}, e^{*} \cdot}$

Proposition 2.2 If $P \phi \in P \Gamma$ and $a_{*} \nsim b^{*}$, then $P \phi$ commutes with $P \zeta_{a_{*}, b^{*}}$ if and only if $P \phi$ fixes $a_{*}$ and $b^{*}$.

Proof. If $P \phi$ fixes $a_{*}$ and $b^{*}$, Proposition 2.1 implies that $P \phi$ commutes with $P \zeta_{a_{*}, b^{*}}$. Conversely, assume that $P \phi$ commutes with $P \zeta_{a_{*}, b^{*}}$ We can assume that $a_{*}=e_{1 *}$ and $b^{*}=e_{1}^{*}$, by Proposition 2.1. $\phi \zeta_{e_{1 *}, e_{1}^{*}} \phi^{-1}=\alpha \zeta_{e_{1 *}, e_{i}^{*}}$ for $\alpha \in R-m . J$ is the direct sum of eigenspaces for $\zeta_{e_{1 *}, e_{i}^{*}}$ of ranks 11 and 16, so $\alpha=1$ and $\phi$ commutes with $\zeta_{e_{1 *}, e_{i}^{*}}$. Thus $\phi$ preserves the 1-eigenspace $R e_{1}+J_{0}\left(e_{1}\right)$ of $\zeta_{e_{1 *}, e_{i}^{*}}$ If $x \in R e_{1}+J_{0}\left(e_{1}\right), x \in J-m J$, and $x^{\#}=0$, it follows that either $x \in R e_{1}$ or $x \in J_{0}\left(e_{1}\right)$. Let $x_{1}=\gamma_{2} e_{2}+1[23]+\gamma_{3} e_{3}, x_{2}=e_{2}$, and $x_{3}=e_{3}$. Since at most one of the $x_{i}$ can satisfy $\phi x_{i} \in R e_{1}$, there are $j \neq k$ such that $\phi x_{j}, \phi x_{k} \in J_{0}\left(e_{1}\right)$. Then

$$
P \phi e_{1}^{*}=P \phi\left(x_{j} \times x_{k}\right)^{*}=\left(\phi x_{j} \times \phi x_{k}\right)^{*}=e_{1}^{*} \text {. }
$$

Since $J_{0}\left(e_{1}\right)=\Sigma R x$ such that $x_{*} \mid e_{1}^{*}, \phi$ preserves $J_{0}\left(e_{1}\right)$. As above, either $\phi e_{1} \in R e_{1}$ or $\phi e_{1} \in J_{0}\left(e_{1}\right)$, since $\phi$ preserves $R e_{1}+J_{0}\left(e_{1}\right)$. Since $\phi$ preserves $J_{0}\left(e_{1}\right), \phi e_{1} \in R e_{1}$. Thus $P \phi$ fixes $e_{1 *}$ and $e_{1}^{*}$.

A subalgebra $Q$ of $\subseteq$ is called a quaternion subalgebra if $Q$ is a free $R$-module of rank 4 and the restriction of $n(x)$ to $Q$ is nondegenerate. $D=Q \oplus Q^{\perp}$ and we define $\tau_{Q} \in \operatorname{End}_{R}(\mathfrak{D})$ to be 1 on $Q$ and -1 on $Q^{\perp} . \tau_{Q}$ is an algebra automorphism of period two, by the proof of [3, Lemma 1.9]. Define an algebra automorphism $\zeta_{Q}$ of $J$ by

$$
\zeta_{Q}\left(\sum \alpha_{i} e_{i}+\sum a_{i}[j k]\right)=\sum \alpha_{i} e_{i}+\sum \tau_{Q}\left(a_{i}\right)[j k] .
$$

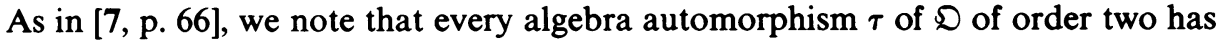
the form $\tau_{Q}$ for a quaternion subalgebra $Q$. To see this, let $Q$ be the 1-eigenspace of $\tau$ and let $P$ be the -1-eigenspace. Since 2 is a unit in $R, D=Q \oplus P$, whence $Q$ and $P$ are free $R$-modules [5, p. 24]. $n(\tau x)=n(x)$ for $x \in J$ [Lemma 1.1]. It follows that $n(P, Q)=0$, so $n$ is nondegenerate on $Q$ and $P$. Since $P \neq 0$, it contains an element whose norm is a unit. Multiplication by this element interchanges $Q$ and $P$, so $Q$ has rank 4. Then $Q$ is a quaternion subalgebra and $\tau=\tau_{Q}$.

Proposition 2.3. Every involution of the second kind is conjugate in $P G$ to $P \zeta_{Q}$ for some quaternion algebra $Q$ of $\$$.

PRoof. Let $P \zeta$ be an involution of the second kind. Let $J_{1}=H\left(\mathscr{D}_{3}, 1\right)$ and define a norm similarity $\psi: J \rightarrow J_{1}$ by $\psi(X)=X \gamma^{-1} . P \psi \zeta \psi^{-1}$ is an involution of the second kind in $P G\left(J_{1}\right)$. There is $\phi \in G\left(J_{1}\right)$ such that $P \phi \psi \zeta \psi^{-1} \phi^{-1}$ fixes $e_{1 *}, e_{2 *}, e_{3 *}$, and $\left(\sum e_{i}+\sum 1[j k]\right)_{*}$, since $P G\left(J_{1}\right)$ is transitive on four-points [3, Lemma 8.1]. The proof of [3, Theorem 8.4] shows that there is a ring automorphism $\tau$ of $\mathfrak{D}$ such that $P \phi \psi \zeta \psi^{-1} \phi^{-1}=P \eta_{\tau}$, where $\eta_{\tau}$ is the norm semisimilarity of $J_{1}$ applying $\tau$ to each 
coordinate. $\eta_{\tau}$ is a scalar multiple of $\phi \psi \zeta \psi^{-1} \phi^{-1}\left[3\right.$, Lemma 3.3], so $\eta_{\tau}$ is linear and $\tau$ is an algebra automorphism. $P \eta_{\tau}^{2}=1$, so $\eta_{\tau}^{2}$ is scalar multiplication. Since $\tau$ fixes $1 \in \mathfrak{D}, \tau^{2}=1$. As above, $\tau=\tau_{Q}$ for a quaternion subalgebra $Q$ of $\mathfrak{D}$. $\eta_{\tau}=\zeta_{Q}$ and

$$
P\left(\psi^{-1} \phi \psi\right) \zeta\left(\psi^{-1} \phi^{-1} \psi\right)=P \psi^{-1} \zeta_{Q} \psi,
$$

where $\psi^{-1} \phi \psi \in G(J)$ and $P \psi^{-1} \zeta_{Q} \psi$ is the involution in $P G(J)$ applying $\tau_{Q}$ coordinatewise.

3. Classification of involutions in $P G$. We prove in this section that every involution in $P G$ is of the first or second kind. (It is immediate that every involution in $P G$ belongs to $P S$, but it is convenient to work in $P G$ since $G$ is closed under scalar multiplication.)

Lemma 3.1. If $R=F$ is a field, there is no involution $P \phi \in P G$ such that $P \phi x_{*} \sim x_{*}$ for all $x_{*} \in P J$.

Proof. Assume such $P \phi$ exists. We claim that $P \phi$ fixes either a point or a line. Suppose that $P \phi$ does not fix $e_{1 *}$. Since $F$ is a field, $P S$ is transitive on pairs of connected points [6, p. 38]. Replacing $P \phi$ by a conjugate, we can assume that $P \phi e_{1 *}=(a[12])_{*}$. Applying $P \phi$ to the equation $(a[12])_{*} \sim e_{2 *}$ gives $e_{1 *} \sim P \phi e_{2 *}$. Since $e_{2 *} \sim P \phi e_{2 *}$ as well, $P \phi e_{2 *}=(b[12])_{*}$. Then

$$
P \phi e_{3 *}=P \phi\left(e_{1} \times e_{2}\right)^{*}=(a[12] \times b[12])^{*}=\left(-\gamma_{1} \gamma_{2} n(a, b) e_{3}\right)^{*},
$$

so $P \phi$ fixes $e_{3 *}$. Thus $P \phi$ fixes a point or a line.

We can assume that either $\phi^{2}=1$ or $\left(\phi^{*-1}\right)^{2}=1$, by the proof of Proposition 2.1. Since $\phi \rightarrow \phi^{*-1}$ is a group isomorphism of $P \Gamma, \phi^{2}=1$ in either case. Since $P \phi e_{1 *} \sim e_{1 *}, \phi e_{1}=\alpha e_{1}+c[12]+d[31]$, where $n(c)=0=n(d)$ and $d c=0$. Set $f_{1}=e_{1}+\phi e_{1}$ and $f_{2}=e_{1}-\phi e_{1} \cdot f_{i}^{\#}=0$ and $\phi f_{i}= \pm f_{i}$. Since $F$ is not of characteristic two, the $e_{1}$ coefficient of at least one of the $f_{i}$ is nonzero. Thus $P \phi$ fixes a point $f_{i *}$ such that the $e_{1}$ coefficient of $f_{i}$ is nonzero.

Replacing $P \phi$ by a conjugate, we can assume that $P_{\phi}$ fixes $e_{2 *}[3$, Proposition 2.1]. Applying the last paragraph again shows that $P \phi$ fixes a point $f_{i *} \nsim e_{2 *}$. Conjugating $P \phi$, we can assume that it fixes $e_{2 *}$ and $e_{3 *}$. Again by the preceding paragraph, $P \phi$ fixes a point $f_{i *}$ such that $\left(f_{i *}, e_{2 *}, e_{3 *}\right)$ is a three-point.

Hence we can assume that $P \phi$ fixes $\left(e_{1 *}, e_{2 *}, e_{3 *}\right)$ and $\phi^{2}=1$. Since $\phi e_{i}= \pm e_{i}$, we can replace $\phi$ by $-\phi$ if necessary and assume that $\phi$ fixes at least two of the $e_{i}$. Replacing $\phi$ by a conjugate, we can assume that it fixes $e_{1}, e_{2}$, and $\mathrm{Fe}_{3}$.

$\phi$ induces $\sigma \in \operatorname{End}_{F}(\mathfrak{D})$ by $\phi(a[12])=a^{\sigma}[12]\left[3\right.$, Lemma 3.2]. $\sigma^{2}=1$, so $\mathfrak{D}=\mathfrak{D}_{1}$ $\oplus \mathfrak{D}_{-1}$ where $\mathfrak{D}_{i}$ is the $i$-eigenspace of $\sigma$. $n\left(a^{\sigma}\right)=n(a)$ for $a \in \mathcal{D}$, since $(\phi x)^{\#}=0$ for $x=e_{1}+a[12]+\gamma_{1} \gamma_{2} n(a) e_{2}$ and $\phi$ fixes $e_{1}$ and $e_{2}$. It follows that the $\mathfrak{D}_{i}$ are orthogonal and the restriction of $n$ to $\mathfrak{D}_{-1}$ is nondegenerate. If $\mathfrak{D}_{-1} \neq 0$, there is $a \in \mathfrak{D}_{-1}$ such that $n(a)$ is nonzero; taking $x=e_{1}+a[12]+\gamma_{1} \gamma_{2} n(a) e_{2}$ gives $x \times$ $\phi x \neq 0$, a contradiction. Thus $\mathfrak{D}_{-1}=0$ and $\phi$ is the identity on $J_{0}\left(e_{3}\right)$. Since $\phi$ fixes $F e_{3}, P \phi=P \zeta_{e_{3 *}, e_{3}^{*}}\left[\right.$ Proposition 2.1]. Then $x_{*} \nsim P \phi x_{*}$ for $x=e_{2}+1[23]+\gamma_{2} \gamma_{3} e_{3}$, a contradiction. 
LEMMA 3.2. There is no involution $P \phi \in P G(J)$ inducing the identity in $P G(J / m J)$.

Proof. Assume such $P \phi$ exists. Only a finite number of elements of $R$ are required to express the multiplication in $J$ and the action of $\phi$ in terms of a given free basis $\left\{x_{i}\right\}$ of $J$. These elements generate a Noetherian subring $R^{\prime}$ of $R$. Replacing $R^{\prime}$ by its localization at $R^{\prime} \cap m$, we can assume that $R^{\prime}$ is local. Replacing $R$ by $R^{\prime}, J$ by $\sum R^{\prime} x_{i}$ and $\phi$ by its restriction, we can assume that $R$ is Noetherian. Tensoring $J$ with the completion of $R$, we can assume that $R$ is complete local Noetherian. The fact that $P \phi$ is an involution is preserved under tensoring, since $P \phi^{2}=1$ if and only if $\phi^{2}$ is multiplication by $\beta \in R-m[3$, Lemma 3.3]. Let $\phi$ induce multiplication by $\alpha_{1} \in R-m$ on $J / m J$. Setting $\alpha_{i+1}=$ $\alpha_{i}+\left(2 \alpha_{i}\right)^{-1}\left(\beta-\alpha_{i}^{2}\right)$ gives $\alpha_{i}^{2} \equiv \beta$ and $\alpha_{i+1} \equiv \alpha_{i}\left(\bmod m^{i}\right)$ by induction. $\alpha=\lim \alpha_{i}$ satisfies $\alpha^{2}=\beta . J$ is the direct sum of eigenspaces for $\pm \alpha$. Since $\phi$ induces multiplication by $\alpha_{1}$ on $J / m J$, Nakayama's Lemma implies that the $\alpha$-eigenspace equals $J[5$, p. 7]. Thus $P \phi=1$, a contradiction.

Lemma 3.3. If $P \phi \in P G$ is an involution, there are $a_{*}$ and $d_{*}$ in $P J$ such that $\left(a_{*} P \phi a_{*}, d_{*}, P \phi d_{*}\right)$ is a four-point.

Proof. $P \phi$ induces an involution $P \phi_{1} \in P G(J / m J)$ [Lemma 3.2]. There is $a_{1 *} \in P(J / m J)$ such that $P \phi_{1} a_{1 *} \nsim a_{1 *}$ [Lemma 3.1]. Since $P S(J / m J)$ is transitive on points and the canonical map $P S(J) \rightarrow P S(J / m J)$ is surjective [3, Corollary 6.5], there is $a_{*} \in P J$ whose image in $P(J / m J)$ is $a_{1 *} a_{*} \nsim P \phi a_{*}$, since $a_{1 *} \nsim$ $P \phi a_{1 *}$. There is a line $b^{*}$ on $a_{*}$ such that $P \phi a_{*} \nsim b^{*}$ (since we can assume that $a_{*}=e_{1 *}$ and $\left.P \phi a_{*}=e_{2 *}\right)$. We repeatedly apply [3, Lemma 8.2] and its dual. $P \phi b^{*} \nsim b^{*}$, since $P \phi a_{*} \mid P \phi b^{*}$ and $P \phi a_{*} \nsim b^{*}$. Let $c_{*}=(b \times \phi b)_{*} c_{*} \nsim a_{*}$, since $a_{*} \nsim P \phi b^{*}$. There is $d_{*} \mid b^{*}$ such that $d_{*} \nsim a_{*}$ and $d_{*} \nsim c_{*}$ (since we can assume that $a_{*}=e_{1 *}$, and $c_{*}=e_{2 *}$, so $\left.b^{*}=e_{3 *}\right) . d_{*} \nsim P \phi b^{*}$, else $P \phi b^{*} \sim(d \times c)^{*}=b^{*}$. $P \phi a_{*} \nsim b^{*}=(a \times d)^{*}$ and $d_{*} \nsim P \phi b^{*}=(\phi a \times \phi d)^{*}$, and applying $P \phi$ gives $a_{*}$ $\nsim(\phi a \times \phi d)^{*}$ and $P \phi d_{*} \nsim(a \times d)^{*}$.

THEOREM 3.4. Every involution $P \phi \in P G$ is of the first or second kind.

Proof. Let $P \phi \in P G$ be an involution. Let

$$
\begin{aligned}
& z_{1}=\gamma_{1} e_{1}+\gamma_{2} e_{2}+1[12], \\
& z_{2}=\gamma_{1} e_{1}+\gamma_{2} e_{2}-1[12], \\
& z_{3}=\gamma_{1} e_{1}+\gamma_{3} e_{3}+1[31], \\
& z_{4}=\gamma_{1} e_{1}+\gamma_{3} e_{3}-1[31], \\
& z_{5}=\gamma_{2} e_{2}+\gamma_{3} e_{3}+1[23], \\
& z_{6}=\gamma_{2} e_{2}+\gamma_{3} e_{3}-1[23]
\end{aligned}
$$

$\left(z_{1} \times z_{2}\right)_{*}=e_{3 *},\left(z_{3} \times z_{4}\right)_{*}=e_{2 *}$, and $\left(z_{5} \times z_{6}\right)_{*}=e_{1 *}$, so $\left(z_{1}^{*}, z_{2}^{*}, z_{3}^{*}, z_{4}^{*}\right)$ is the dual of a four-point. [3, Lemma 8.2] implies that $\left(\left(z_{1} \times z_{3}\right)_{*},\left(z_{1} \times z_{4}\right)_{*},\left(z_{2} \times z_{3}\right)_{*}\right.$, $\left.\left(z_{2} \times z_{4}\right)_{*}\right)$ is a four-point. There are $a_{*}$ and $d_{*}$ in $P J$ such that 
$\left(a_{*}, P \phi a_{*}, d_{*}, P \phi d_{*}\right)$ is a four-point [Lemma 3.3]. Since $P G$ is transitive on fourpoints, we can replace $P \phi$ by a conjugate and assume that these two four-points are equal [3, Lemma 8.1]. Computation shows that $\left(z_{1} \times z_{3}\right) \times e_{1}=\gamma_{1} z_{6}$, so $\left(z_{1} \times z_{3}\right)_{*}$ is on $z_{6}^{*}$, and $z_{1}^{*}, z_{3}^{*}$, and $z_{6}^{*}$ are concurrent. Applying $P \zeta_{e_{3 *}, e_{3}}$ shows that $z_{1}^{*}, z_{4}^{*}$, and $z_{5}^{*}$ are concurrent, applying $P \zeta_{e_{2}, e_{2}^{*}}$ shows that $z_{2}^{*}, z_{3}^{*}$, and $z_{5}^{*}$ are concurrent, and applying $P \zeta_{e_{1}, e_{i}^{*}}$ shows that $z_{2}^{*}, z_{4}^{*}$, and $z_{2}^{*}, z_{4}^{*}$, and $z_{6}^{*}$ are concurrent, as in Figure 1. Let

$$
y_{*}=\left(e_{3} \times z_{2}\right)_{*}=\left(\gamma_{2} e_{1}+\gamma_{1} e_{2}+1[12]\right)_{*} .
$$

$P \phi$ interchanges $a_{*}$ and $d_{*}$ with $P \phi a_{*}$ and $P \phi d_{*}$ respectively, so $P \phi$ interchanges $z_{3}^{*}$ and $z_{5}^{*}$ with $z_{4}^{*}$ and $z_{6}^{*}$ respectively and fixes $z_{1}^{*}$ and $z_{2}^{*}$. Then $P \phi$ fixes all $e_{i^{*}}$ and $e_{i}^{*}$ and hence $y_{*}$. We can assume that $\phi$ fixes $e_{1}$ and that $\phi^{2}=1$, by the proof of Proposition 2.1.

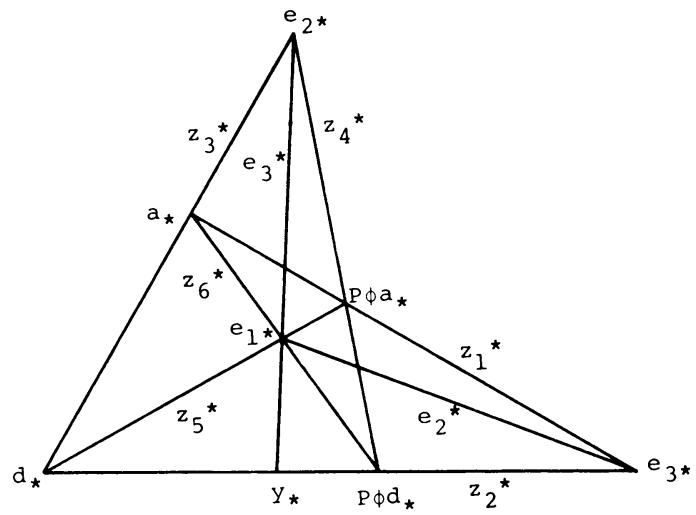

FIGURE 1

Since $\phi$ fixes each $R e_{i}$, it fixes each $\mathscr{D}[j k]\left[3\right.$, Lemma 3.2]. Define $\sigma \in \operatorname{End}_{R}(\mathscr{D})$ by $a^{\sigma}[31]=\phi(a[31]) . \sigma^{2}=1$, since $\phi^{2}=1$. Write $\mathscr{D}=\mathscr{D}_{1} \oplus \mathcal{D}_{-1}$, where $\mathfrak{D}_{i}$ is the $i$-eigenspace of $\sigma . P \phi$ interchanges $a_{*}=\left(z_{1} \times z_{3}\right)_{*}$ with $P \phi a_{*}=\left(z_{1} \times z_{4}\right)_{*}$, where $z_{1} \times z_{3}$ and $z_{1} \times z_{4}$ both have the form $\gamma_{2} \gamma_{3} e_{1}+\gamma_{1} \gamma_{3} e_{2}+\gamma_{1} \gamma_{2} e_{3}+\cdots$. It follows that $\phi$ fixes $e_{2}$ and $e_{3}$ as well as $e_{1}$. Then $\phi$ fixes $1 \in J$, so $\phi \in S$. The equation $N\left(e_{2}+a[31]\right)=-\gamma_{1} \gamma_{3} n(a)$ implies that $n\left(a^{\sigma}\right)=n(a)$ for $a \in \mathcal{D}$. Thus the restrictions of $n$ to $\mathfrak{D}_{1}$ and $\mathfrak{D}_{-1}$ are nondegenerate. If $\mathscr{D}=\mathscr{D}_{-1}, P \phi$ and $P \zeta_{\left.e_{3 *}, e\right\}}$ agree on $e_{2 *}, y_{*}$, and all points on $e_{2}^{*}$. It follows that $P \phi$ equals $P \zeta_{e_{3 *}, e_{3}^{*}}[3$, Lemma 8.3] and $P \phi$ is an involution of the first kind. If $\mathscr{D} \neq \mathscr{D}_{-1}$, there is $x \in D_{1}$ such that $n(x)$ is a unit. Then $\left(e_{2 *}, e_{3 *}, y_{*},\left(e_{1}+x[31]+\gamma_{1} \gamma_{3} n(x) e_{3}\right)_{*}\right)$ is a four-point fixed by $P \phi$, and $P \phi$ is of the second kind.

4. Group-theoretic classification of involutions. We prove now that involutions of the first kind can be distinguished from those of the second kind by their group-theoretic properties within the little projective group. Together with Theorem 
3.4, this implies that an isomorphism of little projective groups preserves each kind of involution.

For $\eta \in S$, let $C(\eta)$ be the centralizer of $\eta$ in $S$ and let $C(P \eta)$ be the centralizer of $P \eta$ in $P S$. Let $P \zeta$ be an involution of the first or second kind and let $P \psi \in C(P \zeta)$. Then $\psi \zeta \psi^{-1}=\alpha \zeta$ for $\alpha \in R-m$. Since $J$ decomposes into eigenspaces of distinct ranks for $\zeta, \alpha=1$ and $\psi \in C(\zeta)$. Hence $C(P \zeta)=P C(\zeta) \simeq$ $C(\zeta) /\left(R^{\times} \cap S\right)$, where $R^{\times}$is the group of units of $R$.

LEMMA 4.1. If $R=F$ is a field and PY is an involution of the first kind, then $C(P \zeta)$ has a normal series where all factor groups are abelian except for one which is simple.

Proof. We can assume that $\zeta=\zeta_{e_{1 *}, e_{1}^{*}}$ [Proposition 2.1]. Since $C(P \zeta) \cong$ $C(\zeta) /\left(F^{\times} \cap S\right)$, it suffices to prove that $C(\zeta)$ has such a normal series. If $\psi \in S$, $\psi \in C(\zeta)$ if and only if $\psi$ preserves $F e_{1}$ and $J_{0}\left(e_{1}\right)$, by the proof of Proposition 2.2. Let $N$ be the kernel of the homomorphism from $C(\zeta)$ to $F^{\times}$taking $\psi$ to $\alpha$ such that $\psi e_{1}=\alpha e_{1} . C(\zeta) / N$ is abelian. Let $\mathscr{D}\left(J_{0}\right)$ be the orthogonal group of $J_{0}\left(e_{1}\right)$ relative to the quadratic form $x \rightarrow T\left(x^{\#}, e_{1}\right)$. Since elements of $S$ preserve $T\left(x^{\sharp}, y\right)[6, \mathrm{p}$. 10], we can define a homomorphism $\lambda: N \rightarrow \Im\left(J_{0}\right)$ by restriction. The kernel of $\lambda$ is $\{1, \zeta\}$ [3, Lemma 5.1]. The image of $\lambda$ is the reduced orthogonal group $\mathfrak{D}^{\prime}\left(J_{0}\right)[6, \mathrm{p}$. 31]. $\mathfrak{D}^{\prime}\left(J_{0}\right)$ modulo its center is simple, since $J_{0}\left(e_{1}\right)$ is an isotropic space of dimension 10 [1, pp. 195 and 209].

LemMA 4.2. If $R=F$ is a field with more than three elements and $P \zeta$ is an involution of the second kind, then $C(P \zeta)$ has a normal series with two nonsolvable factor groups.

Proof. We can assume that $\gamma=1$ and that $P \zeta=P \zeta_{Q}$ for a quaternion subalgebra $Q$ of $\mathfrak{D}$ [Theorem 1.3 and Proposition 2.3]. Since $C\left(P \zeta_{Q}\right) \simeq C\left(\zeta_{Q}\right) /\left(F^{\times} \cap S\right)$, it suffices to prove that $C\left(\zeta_{Q}\right)$ has a normal series with two nonsolvable factor groups. $C\left(\zeta_{Q}\right)$ consists of the elements of $S$ preserving the \pm 1 -eigenspaces of $\zeta_{Q}$. Identify the 1-eigenspace of $\zeta_{Q}$ with $H\left(Q_{3}, \pi\right)$, where $\pi$ is the standard involution conjugate transpose of $Q_{3}$. Let $\tau$ be the restriction homomorphism from $C\left(\zeta_{Q}\right)$ to $\operatorname{End}_{F}\left(H\left(Q_{3}, \pi\right)\right)$. We claim that neither the kernel nor the image of $\tau$ is solvable. If $Q$ is a division algebra, we are done by the proof of [10, pp. 333-334]. Assume that $Q$ is split, so $Q \cong F_{2}[8$, p. 169].

The proof of $[10$, p. 334] shows that the kernel of $\tau$ is isomorphic to the group of elements of $Q$ of norm 1. This group is isomorphic to $\mathrm{SL}_{2}(F)$, which is not solvable if $F$ has more than three elements [1, p. 169].

To see that the image of $\tau$ is not solvable, let $T=T_{x[i j], e,}$ for $x \in Q, i \neq j$. $T \in S(J)$ [6, p. 18]. $T \in C\left(\zeta_{Q}\right)$, since $\zeta_{Q}$ is an automorphism fixing $x[i j]$ and $e_{j}$. Define a homomorphism $\lambda$ from the group of invertible elements of $Q_{3}$ to the group of invertible linear transformations of $H\left(Q_{3}, \pi\right)$ by $\lambda(A)=A X A^{\pi}, A \in Q_{3}$, $X \in H\left(Q_{3}, \pi\right) . \tau(T)=\lambda\left(1+x e_{i j}\right)$, where the $e_{i j}$ are matrix units decomposing $Q_{3}$ over $Q$. Let $W$ be the multiplicative subgroup of $Q_{3}$ generated by $1+x e_{i j}, x \in Q$, $i \neq j$. Identify $Q \cong F_{2}$ and let the matrix units of $F_{2}$ be written as $f_{s t}$. If $\alpha \in F$, $s \neq t$ and $i \neq j, W$ contains

$$
\left(1+e_{i j}\right)\left(1-f_{t t} e_{j i}\right)\left(1+\alpha f_{s t} e_{i j}\right)\left(1+f_{t t} e_{j i}\right)\left(1-e_{i j}\right)=1+\alpha f_{s t} e_{i i}
$$


Thus $W$ contains $1+\alpha f_{s t} e_{i j}, \alpha \in F$, if either $s \neq t$ or $i \neq j$. Let $\eta$ be the natural isomorphism of $\left(F_{2}\right)_{3}$ onto $F_{6}$ and let the matrix units of $F_{6}$ be written $g_{u v}$. Then $\eta(W)$ contains $1+\alpha g_{u v}$ for all $\alpha \in F$ and $u \neq v$. These elements generate $\operatorname{SL}_{6}(F)$ $\left[1\right.$, p. 156], so $W=\eta^{-1}\left(\mathrm{SL}_{6}(F)\right)$. One verifies that the kernel of $\lambda$ is \pm 1 . Since $\operatorname{PSL}_{6}(F)$ is simple [1, p. 169], $\lambda(W)$ is not solvable. Since $\lambda\left(1+x e_{i j}\right)=\tau(T), \lambda(W)$ is contained in $\tau\left(C\left(\zeta_{Q}\right)\right)$ and $\tau\left(C\left(\zeta_{Q}\right)\right)$ is not solvable.

Let $P H_{i}(J)$ be the set of involutions of the $i$ th kind in $P S(J)$.

LEMMA 4.3. Let $\theta$ be an isomorphism of $P S(J)$ onto $P S\left(J^{\prime}\right)$, where $R=F$ and $R^{\prime}=F^{\prime}$ are fields. Then $\theta\left(P H_{i}(J)\right)=P H_{i}\left(J^{\prime}\right)$.

Proof. Since $P H_{1}(J)$ is a conjugacy class of $P S(J)$, it suffices to prove that either $\theta\left(P H_{1}(J)\right) \subset P H_{1}\left(J^{\prime}\right)$ or $\theta^{-1}\left(P H_{1}\left(J^{\prime}\right)\right) \subset P H_{1}(J)$. We are done by Theorem 3.4 and Lemmas 4.1 and 4.2, unless both $F$ and $F^{\prime}$ have three elements. Assume that is the case. If $P \zeta_{1} \in P H_{1}(J)$, the proof of Lemma 4.1 shows that $C\left(\zeta_{1}\right)$ has a normal subgroup $N$ of index at most two such that $N /\left\{1, \zeta_{1}\right\} \simeq \mathfrak{D}^{\prime}\left(J_{0}\right)$ and $\mathfrak{D}^{\prime}\left(J_{0}\right)$ modulo its center is simple. The center of $\mathfrak{D}^{\prime}\left(J_{0}\right)$ has order at most two [1, p. 133]. Let $Q^{\prime}$ be a quaternion subalgebra of $\mathfrak{D}^{\prime}$. Since $F^{\prime}$ is finite, $Q^{\prime}$ is split [1, p. 144]. The second paragraph of the proof of Lemma 4.2 shows that $C\left(\zeta_{Q^{\prime}}\right)$ has a normal subgroup isomorphic to $\mathrm{SL}_{2}\left(F^{\prime}\right) . \mathrm{SL}_{2}\left(F^{\prime}\right)$ is solvable of order $24\left[1\right.$, p. 170], so $C\left(\zeta_{1}\right)$ is not isomorphic to $C\left(\zeta_{Q^{\prime}}\right) . C\left(P \zeta_{1}\right) \cong C\left(\zeta_{1}\right)$ and $C\left(P \zeta_{Q^{\prime}}\right) \cong C\left(\zeta_{Q^{\prime}}\right)$, since $F^{\times} \cap S$ $=\left\{\alpha \in F \mid \alpha^{3}=1\right\}=\{1\}$. Thus $C\left(P \zeta_{1}\right)$ is not isomorphic to $C\left(P \zeta_{Q^{\prime}}\right)$. If $P \zeta_{2} \in$ $P H_{2}(J)$, there is $\psi \in G$ such that $P \psi \zeta_{2} \psi^{-1}=P \zeta_{Q^{\prime}}$ for some quaternion subalgebra $Q^{\prime}$ of $\mathfrak{D}^{\prime}$ [Proposition 2.3], so $C\left(P \zeta_{2}\right) \cong C P\left(\zeta_{Q^{\prime}}\right)$. Thus $C\left(P \zeta_{1}\right)$ and $C\left(P \zeta_{2}\right)$ are not isomorphic. We are done by Theorem 3.4.

THEOREM 4.4. If $\theta$ is an isomorphism of $P S(J)$ onto $P S\left(J^{\prime}\right)$, then $\theta\left(P H_{i}(J)\right)=$ $P H_{i}\left(J^{\prime}\right)$.

Proof. The canonical homomorphism from $P S(J)$ to $P S(J / m J)$ is surjective [3, Corollary 6.5]. Let $P S_{m}(J)$ be its kernel, so $P S(J) / P S_{m}(J) \simeq P S(J / m J)$. $\theta\left(P S_{m}(J)\right)=P S_{m^{\prime}}\left(J^{\prime}\right)$, since $P S_{m}(J)$ is the unique maximal normal subgroup of $P S(J)$ [3, Corollary 7.5]. Thus $\theta$ induces an isomorphism $\theta_{m}$ of $P S(J / m J)$ onto $P S\left(J^{\prime} / m^{\prime} J^{\prime}\right)$. By Lemma 4.3, $\theta_{m}\left(P H_{i}(J / m J)\right)=P H_{i}\left(J^{\prime} / m^{\prime} J^{\prime}\right)$. It follows from Theorem 3.4 that $\theta\left(P H_{i}(J)\right)=P H_{i}\left(J^{\prime}\right)$.

5. Commuting involutions of the first kind. We prove in this section $P \zeta_{a_{*}, b^{*}}$ and $P \zeta_{c_{*}, d^{*}}$ commute and their product is an involution of the first kind if and only if $a_{*} \mid d^{*}$ and $c_{*} \mid b^{*}$. The key step is to characterize the action of involutions of the first kind in terms of the harmonic properties of the plane.

Lemma 5.1. $P \zeta_{e_{1 *}, e_{i}^{*}}$ commutes with $P \zeta_{a_{*}, b^{*}}$ if and only if one of the following conditions holds:

(1) $a_{*}=e_{1 *}$ and $b^{*}=e_{1}^{*}$,

(2) $a_{*} \mid e_{1}^{*}$ and $e_{1 *} \mid b^{*}$,

(3) $a, b \in J_{1 / 2}\left(e_{1}\right)$. 
Proof. Write $e_{1 *}$ as $e_{*} . P \zeta_{e_{*}, e^{*}}$ commutes with $P \zeta_{a_{*}, b^{*}}$ if and only if $P \zeta_{e_{*}, e^{*}}$ fixes $a_{*}$ and $b^{*}$ [Proposition 2.2]. This holds if and only if $\zeta_{e_{*}, e^{*}}$ fixes $R a$ and $R b$, since one checks that $\zeta_{e_{*}^{*}, e^{*}}^{*-1}=\zeta_{e_{*}, e^{*}}$. This is equivalent to assuming that $a$ and $b$ are elements of $R e+J_{0}(e)$ or $J_{1 / 2}(e)$, the eigenspaces of $\zeta_{e_{*}, e^{*}}$. This holds if and only if $a$ and $b$ are elements of $R e, J_{0}(e)$, and $J_{1 / 2}(e)$, by the proof of Proposition 2.2. The lemma follows, since $a_{*} \nsim b^{*}$ and the spaces $R e, J_{0}(e)$, and $J_{1 / 2}(e)$ are orthogonal with respect to $T(x, y)$.

LEMMA 5.2. If $a_{*} \nsim b^{*}, c_{*} \nsim d^{*}, a_{*} \mid d^{*}$, and $c_{*} \mid b^{*}$, then

$$
P \zeta_{a_{*}, b^{*}} P \zeta_{c_{*}, d^{*}}=P \zeta_{(b \times d)_{*},(a \times c)^{*}}
$$

and $\left(a_{*}, c_{*},(b \times d)_{*}\right)$ is a three-point.

Proof. $a_{*} \nsim b^{*}$ implies that $b^{*} \nsim d^{*}$ and $a_{*} \nsim(b \times d)_{*}$, by [3, Lemma 8.2] and its dual. Then $(a \times(b \times d))^{*}=d^{*}$, so $c_{*} \nsim d^{*}$ implies that $\left(a_{*}, c_{*},(b \times d)_{*}\right)$ is a three-point. We can assume that this three-point equals $\left(e_{1 *}, e_{2 *}, e_{3 *}\right)$ [3, Proposition 2.1]. Then $b^{*}=(c \times(b \times d))^{*}=e_{1}^{*}$ and $d^{*}=(a \times(b \times d))^{*}=e_{2}^{*}$. Since $\zeta_{e_{i *}, e_{i}^{*}}$ is 1 on $R e_{i}+J_{0}\left(e_{i}\right)$ and -1 on $J_{1 / 2}\left(e_{i}\right), P \zeta_{e_{1 *}, e_{i}^{*}} P \zeta_{e_{2 *}, e_{2}^{*}}=P \zeta_{e_{3 *}, e_{3}^{*}}$. The lemma follows, since $a_{*}=e_{1 *}, b^{*}=e_{1}^{*}, c_{*}=e_{2 *}$, and $d^{*}=e_{2}^{*}$.

When $R=F$ is a field, if $x_{*} x_{*}$ and $w_{*} \mid(x \times y)^{*}$, Faulkner has defined the harmonic conjugate of $w_{*}$ with respect to $x_{*}$ and $y_{*}[6$, p. 42]. We remark that his results and the following lemma extend directly to octonion planes over local rings, but we do not need this extension.

LEMMA 5.3. If $R=F$ is a field, $a_{*} \nsim b^{*}$, and $a_{*} \mid d^{*}$, then $P \zeta_{a_{*}, b^{*}}$ takes every point on $d^{*}$ to its harmonic conjugate with respect to $a_{*}$ and $(b \times d)_{*}$.

Proof. $d^{*} \nsim b^{*}$ and $a_{*} \nsim(b \times d)_{*}$, since $a_{*} \nsim b^{*}\left[6\right.$, p. 36]. There is $f_{*} \mid b^{*}$ such that $f_{*} \nsim(b \times d)_{*}\left[6\right.$, p. 36]. $a_{*} \nsim b^{*}=((b \times d) \times f)^{*}$, so $\left(a_{*},(b \times d)_{*}, f_{*}\right)$ is a three-point, and we can assume that it equals $\left(e_{1 *}, e_{2 *}, e_{3 *}\right)[6$, p. 33]. It follows that $b^{*}=e_{1}^{*}$ and $d^{*}=e_{3}^{*}$. The harmonic conjugate of $\left(\alpha_{1} e_{1}+s[12]+\alpha_{2} e_{2}\right)_{*}$ with respect to $e_{1 *}$ and $e_{2 *}$ is $\left(\alpha_{1} e_{1}-s[12]+\alpha_{2} e_{2}\right)_{*}\left[6\right.$, p. 42]. Then $P \zeta_{e_{1 *}, e_{i}^{*}}$ takes every point on $e_{3}^{*}$ to its harmonic conjugate with respect to $e_{1 *}$ and $e_{2 *}$ as required.

Lemma 5.4. Assume that $R=F$ is a field, $a_{*} \nsim b^{*}$, and $a, b \in J_{1 / 2}\left(e_{1}\right)$. Then $P \zeta_{e_{1}, e_{1}^{*}} P \zeta_{a_{*}, b^{*}}$ is not an involution of the first kind.

Proof. Since $F$ is a field, there is a line $f^{*}$ on $e_{1 *}$ and $a_{*}\left[6\right.$, p. 35]. $f^{*} \nsim e_{1}^{*}$, since $e_{1 *} \mid f^{*}$ and $e_{1 *} \nsim e_{1}^{*}\left[6\right.$, p. 36]. Take $g_{*} \mid e_{1}^{*}$ such that $g_{*} \nsim\left(f \times e_{1}\right)_{*} \cdot e_{1 *} \nsim e_{1}^{*}=$ $\left(\left(f \times e_{1}\right) \times g\right)^{*}$, so there is $P \phi \in P S$ taking the three-point $\left(e_{1 *},\left(f \times e_{1}\right)_{*}, g_{*}\right)$ to $\left(e_{1 *}, e_{2 *}, e_{3 *}\right)$. Since $\left(\left(f \times e_{1}\right) \times g\right)^{*}=e_{1}^{*}, P \phi$ fixes $e_{1 *}$ and $e_{1}^{*}$. Lemma 5.1 implies that $\phi a, \phi b \in J_{1 / 2}\left(e_{1}\right)$. Thus we can replace $P \zeta_{a_{*}, b^{*}}$ with its conjugate by $P \phi$. Then $f^{*}=\left(e_{1} \times\left(f \times e_{1}\right)\right)^{*}=e_{3}^{*}$, so $a_{*} \mid e_{3}^{*}$.

$e_{3}^{*} \nsim b^{*}$, since $a_{*} \mid e_{3}^{*}$ and $a_{*} \nsim b^{*}$. $a$ and $e_{3} \times b$ are in $J_{1 / 2}\left(e_{1}\right) \cap J_{0}\left(e_{3}\right)$, so $a_{*}=(s[12])_{*}$ and $\left(e_{3} \times b\right)_{*}=(t[12])_{*}$ for $n(s)=0=n(t) . a_{*} \nsim b^{*}$ implies that $a_{*} \nsim\left(e_{3} \times b\right)_{*}$, so $n(s, t)$ is a unit. Replace $t$ by a scalar multiple to make $n(s, t)=$ $-\gamma_{1}^{-1} \gamma_{2}^{-1}$. 
Let $W$ be the orthogonal complement of $F s+F t$ in $\mathfrak{D}$ with respect to $n(x, y)$. Define a linear transformation $\psi$ on $J_{0}\left(e_{3}\right)$ to interchange $e_{1}$ and $e_{2}$ with $s[12]$ and $t[12]$ respectively and to be the identity on $W[12] \cdot \psi^{2}=1$ and $\psi$ belongs to the orthogonal group of the quadratic form $T\left(x^{\#}\right)$ on $J_{0}\left(e_{3}\right)$. Since its determinant is 1 , $\psi$ is the product of an even number of hyperplane reflections [1, p. 129]. Then $\psi$ is induced by an element $\eta$ of $G$ preserving $F e_{3}$ [3, Theorem 5.3]. $P \zeta_{a_{*}, b^{*}}$ takes every point on $e_{3}^{*}$ to its harmonic conjugate with respect to $a_{*}$ and $\left(e_{3} \times b\right)^{*}$ [Lemma 5.3]. Likewise $P \zeta_{e_{1 *}, e_{i}^{*}}$ takes every point on $e_{3}^{*}$ to its harmonic conjugate with respect to $e_{1 *}$ and $e_{2 *}$. It follows that $P \zeta_{a_{*}, b^{*}}$ agrees with $P \eta \zeta_{e_{1 *}, e_{1}^{*}} \eta^{-1}$ on all points on $e_{3}^{*}$. Let

$$
x_{*}=\left(\alpha e_{1}+\beta e_{2}+(\delta s+\lambda t+w)[12]\right)_{*}
$$

where $\alpha, \beta, \delta, \lambda \in F, w \in W$, and $x^{\#}=0$. Then

$$
\begin{aligned}
& P \zeta_{a_{*}, b^{*}}\left(x_{*}\right)=\left(\psi \zeta_{e_{1 *}, e_{1}^{*}} \psi x\right)_{*} \\
&=\left(-\alpha e_{1}-\beta e_{2}+(\delta s+\lambda t-w)[12]\right)_{*} \\
& P \zeta_{e_{1 *}, e_{1}^{*}} P \zeta_{a_{*}, b^{*}}\left(x_{*}\right)=\left(-\alpha e_{1}-\beta e_{2}+(-\delta s-\lambda t+w)[12]\right)_{*}
\end{aligned}
$$

Take a preimage of $P \zeta_{e_{1 *}, e_{i}^{*}} P \zeta_{a_{*}, b^{*}}$ in $G$ and let $\tau$ be its restriction to $J_{0}\left(e_{3}\right)$. (2) implies that $\tau$ is a scalar multiple of the map which is 1 on $W[12]$ and -1 on $W[12]^{\perp}$. Thus $\tau$ has eigenspaces of dimensions 4 and 6 .

Assume that $P \zeta_{e_{1 *}, e_{i}^{*}} P \zeta_{a_{*}, b^{*}}=P \zeta_{c_{*}, d^{*}}$. It is not true that $c_{*} \mid e_{1}^{*}$ and $e_{1 *} \mid d^{*}$, else Lemma 5.2 would imply that $a_{*} \mid e_{1}^{*}$ and $e_{1 *} \mid b^{*}$. Since $P \zeta_{e_{1_{*}}, e_{1}^{*}}$ and $P \zeta_{c_{*}, d^{*}}$ commute, Lemma 5.1 implies that $c, d \in J_{1 / 2}\left(e_{1}\right)$. Take $v \in W$ such that $n(v) \neq 0$, and set

$$
y_{*}=\left(e_{1}+\gamma_{1} \gamma_{2} n(v) e_{2}+v[12]\right)_{*}
$$

(2) shows that $(y \times \tau y)^{*}=e_{3}^{*}$. Since $F$ is a field, there is a line on $c_{*}$ and $y_{*}[6, \mathrm{p}$. 35]. Then Lemma 5.3 and the equation $(y \times \tau y)^{*}=e_{3}^{*}$ imply that $c_{*}$ is on $e_{3}^{*}$. Thus we can apply the analogue of equation (1) for $P \zeta_{c_{*}, d^{*}}$. Take a preimage of $P \zeta_{c_{*}, d^{*}}$ in $G$ and let $\xi$ be its restriction to $J_{0}\left(e_{3}\right)$. (1) implies that $\xi$ has eigenspaces of dimensions 2 and 8 , contradicting the fact that $\xi$ is a scalar multiple of $\tau$.

THEOREM 5.5. If $a_{*} \nsim b^{*}$ and $c_{*} \nsim d^{*}$, the following conditions are equivalent:

(1) $P \zeta_{a_{*}, b^{*}}$ and $P \zeta_{c_{*}, d^{*}}$ commute and their product is an involution of the first kind.

(2) $a_{*} \mid d^{*}$ and $c_{*} \mid b^{*}$.

Proof. (1) $\Rightarrow(2)$. By conjugation, we can assume that $c_{*}=e_{1 *}$ and $d^{*}=e_{1}^{*}$. By Lemma 5.1, either $a_{*} \mid e_{1}^{*}$ and $e_{1 *} \mid b^{*}$ or $a, b \in J_{1 / 2}\left(e_{1}\right)$. Taking images in $P S(\mathrm{~J} / \mathrm{mJ})$ and applying Lemma 5.4 shows that the latter condition does not hold. $(2) \Rightarrow(1)$, by Lemmas 5.1 and 5.2.

We say that $P \zeta_{a_{*}, b^{*}}$ and $P \zeta_{c_{*}, d^{*}}$ commute exactly if the conditions of Theorem 5.5 are satisfied.

COROllary 5.6. $a_{*} \nsim c_{*}$ if and only if there exist $b^{*}$ and $d^{*}$ such that $P \zeta_{a_{*}, b^{*}}$ and $P \zeta_{c_{*}, d^{*}}$ commute exactly. 
Proof. If $P \zeta_{a_{*}, b^{*}}$ and $P \zeta_{c_{*}, d^{*}}$ commute exactly, then $a_{*} \nsim c_{*}$ [Lemma 5.2]. Conversely, if $a_{*} \nsim c_{*}$, we can assume that $a_{*}=e_{1 *}$ and $c_{*}=e_{2 *}$ [3, Proposition 2.1], so we can take $b^{*}=e_{1}^{*}$ and $d^{*}=e_{2}^{*}$.

Corollary 5.7. $a_{*} \mid d^{*}$ if and only if there exist $b^{*}$ and $c_{*}$ such that $P \zeta_{a_{*}, b^{*}}$ and $P \zeta_{c_{*}, d^{*}}$ commute exactly.

Proof. If $a_{*} \mid d^{*}$, there is $g_{*} \mid d^{*}$ such that $a_{*} \nsim g_{*}$ [3, dual of Lemma 2.3]. We can assume that $a_{*}=e_{1 *}$ and $g_{*}=e_{3 *}$, so $d^{*}=e_{2}^{*}$. It suffices to take $b^{*}=e_{1}^{*}$ and $c_{*}=e_{2 *}$. The converse is trivial.

6. Classification of isomorphisms of collineation groups. Let $P H$ be a subgroup of $P G(J)$ containing $P S(J)$ and let $P H^{\prime}$ be a subgroup of $P G\left(J^{\prime}\right)$ containing $P S\left(J^{\prime}\right)$. We prove that every isomorphism of $P H$ onto $P H^{\prime}$ has the form $P \phi \rightarrow P \tau P \phi P \tau^{-1}$ where $P \tau: P J \rightarrow P J^{\prime}$ is a collineation or a correlation.

LeMma 6.1. Let $a_{*}, b^{*}, c^{*} \in P J$ be such that $a_{*} \nsim b^{*}, a_{*} \nsim c^{*}$, and $b^{*} \sim c^{*}$. Then there is $d^{*} \in P J$ such that $a_{*} \nsim d^{*}, b^{*} \nsim d^{*}$, and $c^{*} \nsim d^{*}$.

Proof. We can assume that $a_{*}=e_{1 *}$ and $b^{*}=e_{1}^{*}$, as in $\$ 2$. Since $b^{*} \sim c^{*}$, $c \equiv \alpha e_{1}+x[12]+y[31](\bmod m J)$. Since $a_{*} \nsim c^{*}, \alpha$ is a unit. There is $z \in \mathfrak{D}$ such that $n(x, z) \in m$ and $n(z)$ is a unit. It suffices to set $d=e_{1}+z[12]+\gamma_{1} \gamma_{2} n(z) e_{2}$, since the coefficient of $e_{3}$ in $c \times d$ is a unit.

Proposition 6.2. Let $\theta$ be an isomorphism of $P S(J)$ onto $P S\left(J^{\prime}\right)$. Assume that there are $a_{*}, b^{*}, c^{*} \in P J$ such that

(1) $a_{*} \nsim b^{*}, a_{*} \nsim c^{*}$ and there is a point $d_{*}$ on both $b^{*}$ and $c^{*}$, and

(2) $\theta P \zeta_{a_{*}, b^{*}}=P \zeta_{s_{*}, t^{*}}$ and $\theta P \zeta_{a_{*}, c^{*}}=P \zeta_{u_{*}, v^{*}}$, where $t^{*} \nsim v^{*}$.

Then there is a collineation $P \tau^{*}: P J \rightarrow P J^{\prime}$ such that $\theta P \zeta_{x_{*} y^{*}}=P \tau P \zeta_{x_{*} y^{*}} P \tau^{-1}$ for all $x_{*} \nsim y^{*}$.

Proof. Let $f_{*}, g^{*}, h^{*} \in P J$ satisfy $f_{*} \nsim g^{*}, f_{*} \nsim h^{*}$, and $g^{*} \nsim h^{*}$. Let $\theta P \zeta_{f_{*}, g^{*}}=$ $P \zeta_{n_{*}, p^{*}}$ and $\theta P \zeta_{f_{*}, h^{*}}=P \zeta_{q_{*}, r^{*}}$ [Theorem 4.4]. We claim that $n_{*}=q_{*} f_{*} \nsim(g \times h)_{*}$, since $f_{*} \nsim g^{*}$. $a_{*} \nsim d_{*}$, since $a_{*} \nsim b^{*}$. Thus there is $P \phi \in P S(J)$ such that $P \phi a_{*}=(g \times h)_{*}$ and $P \phi d_{*}=f_{*} \cdot P \zeta_{f_{*}, g^{*}}$ and $P \zeta_{f_{*}, h^{*}}$ commute exactly with $P \zeta_{P \phi a_{*}, P \phi b^{*}}$ and $P \zeta_{P \phi a_{*}, P \phi c^{* *}}$ Applying $\theta$ shows that $P \zeta_{n_{*} p^{*}}$ and $P \zeta_{q_{*}, r^{*}}$ commute exactly with $\theta P \zeta_{P \phi a_{*}, P \phi b^{*}}$ and $\theta P \zeta_{P \phi a_{*}, P \phi c_{*}}$ [Theorems 4.4 and 5.5]. Setting $P \psi=$ $\theta P \phi \in P S\left(J^{\prime}\right)$ gives

$$
\theta P \zeta_{P \phi a_{*}, P \phi b^{*}}=\theta\left(P \phi P \zeta_{a_{*}, b^{*}} P \phi^{-1}\right)=P \psi P \zeta_{s_{*}, t^{*}} P \psi^{-1}=P \zeta_{P \psi s_{*}, P t^{*}}
$$

and likewise $\theta P \zeta_{P \phi a_{*}, P \phi c^{*}}=P \zeta_{P \psi u_{*}, P \psi v^{*}}$ Thus $P \zeta_{n_{*}, p^{*}}$ and $P \zeta_{q_{*}, r^{*}}$ commute exactly with $P \zeta_{P \psi_{*}, P t^{*}}$ and $P \zeta_{P \psi u_{*} P v^{*}}$, so $n_{*}$ and $q_{*}$ are on $P \psi t^{*}$ and $P \psi v^{*}$. By hypothesis, $t^{*} \nsim v^{*}$, so $n_{*}=(\psi t \times \psi v)_{*}=q_{*}$, as asserted.

We have shown that if $f_{*} \nsim g^{*}, f_{*} \nsim h^{*}$, and $g^{*} \nsim h^{*}$, then $\theta P \zeta_{f_{*}, g^{*}}=P \zeta_{n_{*} p^{*}}$ and $\theta P \zeta_{f_{*}, h^{*}}=P \zeta_{n_{*}, r^{*}}$ Lemma 6.1 implies that this remains true without the hypothesis that $g^{*} \nsim h^{*}$. Thus there is a bijection $P \tau_{1}$ from the points of $P J$ onto the points of $P J^{\prime}$ such that $\theta P \zeta_{x_{*} y^{*}}=P \zeta_{P \tau_{1} x_{*} p^{*}}$ for all $x_{*} \nsim y^{*} . x_{1 *} \nsim x_{2 *}$ if and only if $P \tau_{1} x_{1 *} \nsim P \tau_{1} x_{2 *}$ [Corollary 5.6]. 
Take $a^{*}, b_{*}, c_{*} \in P J$ such that $b_{*} \nsim a^{*}, c_{*} \nsim a^{*}$, and $b_{*} \nsim c_{*} \cdot \theta P \zeta_{b_{*}, a^{*}}=$ $P \zeta_{P \tau_{1} b_{*} s^{*}}$ and $\theta P \zeta_{c_{*}, a^{*}}=P \zeta_{P \tau_{1} c_{*}, u^{*}}$, where $P \tau_{1} b_{*} \nsim P \tau_{1} c_{*}$. Thus $\theta$ satisfies the duals of conditions (1) and (2). By duality, there is a bijection $P \tau_{2}$ from the lines of $P J$ onto the lines of $P J^{\prime}$ such that

$$
\theta P \zeta_{x_{*} y^{*}}=P \zeta_{P \tau_{1} x_{*}, P \tau_{2} y^{*}}
$$

for all $x_{*} \nsim y^{*}$; also $y_{1}^{*} \nsim y_{2}^{*}$ if and only if $P \tau_{2} y_{1}^{*} \nsim P \tau_{2} y_{2}^{*}$. By Corollary 5.7, $x_{*} \mid y^{*}$ if and only if $P \tau_{1} x_{*} \mid P \tau_{2} y^{*} . x_{*} \nsim y^{*}$ if and only if $P \zeta_{x_{*} y^{*}}$ is defined, so $x_{*} \nsim y^{*}$ if and only if $P \tau_{1} x_{*} \nsim P \tau_{2} y^{*}$. Thus $x_{*} \rightarrow P \tau_{1} x_{*}$ and $y^{*} \rightarrow P \tau_{2} y^{*}$ define a collineation $P \tau$. We are done by (3).

A correlation of two octonion planes consists of bijections between the points of each plane and the lines of the other preserving the relations "on" and "connected to". Let $P \psi$ be the canonical correlation of $P J^{\prime}$ interchanging $x_{*}$ and $x^{*}$. If $P \phi \in P \Gamma\left(J^{\prime}\right), P \psi P \phi P \psi=P \phi^{*-1}$. If $x_{*} \nsim y^{*}$ in $P J^{\prime}, P \psi P \zeta_{x_{*} y^{*}} P \psi=P \zeta_{y_{*}, x^{*}}$ Every correlation of $P J$ onto $P J^{\prime}$ has the form $P \psi P \eta$ where $\eta$ is a norm semisimilarity of $J$ onto $J^{\prime}[3$, Theorem 8.4].

Proposition 6.3. Let $\theta$ be an isomorphism of PS(J) onto PS $\left(J^{\prime}\right)$. Assume that there are $a_{*}, b^{*}, c^{*} \in P J$ such that

(1) $a_{*} \nsim b^{*}, a_{*} \nsim c^{*}$, and there is a point $d_{*}$ on both $b^{*}$ and $c^{*}$, and

(2) $\theta P \zeta_{a_{*}, b^{*}}=P \zeta_{s_{*}, r^{*}}$ and $\theta P \zeta_{a_{*}, c^{*}}=P \zeta_{u_{*}, v^{*}}$, where $s_{*} \nsim u_{*}$.

Then there is a correlation $P \tau^{*}: P J \rightarrow P J^{\prime}$ such that $\theta P \zeta_{x_{*}, y^{*}}=P \tau P \zeta_{x_{*}, y^{*}} P \tau^{-1}$ for all $x_{*} \nsim y^{*}$.

Proof. Conjugation by the canonical correlation $P \psi$ of $P J^{\prime}$ induces an automorphism $\Psi$ of $P S\left(J^{\prime}\right) .\left(\phi \in S\right.$ implies $\phi^{*-1} \in S$, by the proof of [3, Lemma 1.7].) $\Psi \theta$ satisfies the hypotheses of Proposition 6.2, so there is a collineation $P \eta: P J \rightarrow P J^{\prime}$ such that $\Psi \phi\left(P \zeta_{x_{*} y^{*}}\right)=P \eta P \zeta_{x_{*} y^{*}} P \eta^{-1}$ for all $x_{*} \nsim y^{*}$. Set $P \tau=P \psi P \eta$.

Proposition 6.4. Let $\theta$ be an isomorphism of PS(J) onto $P S\left(J^{\prime}\right)$, where $R=F$ and $R^{\prime}=F^{\prime}$ are fields. Then there is a collineation or a correlation $P \tau: P J \rightarrow P J J^{\prime}$ such that $\theta P \zeta_{x_{*} y^{*}}=P \tau P \zeta_{x_{*} y^{*}} P \tau^{-1}$ for all $x_{*} \nsim y^{*}$.

Proof. Take $a_{*}, b^{*} \in P J$ such that $\theta P \zeta_{a_{*}, b^{*}}=P \xi_{e_{1 *}, e_{i}^{*}}$ [Theorem 4.4]. There is $c^{*} \in P J$ such that $a_{*} \nsim c^{*}$ and $b^{*} \nsim c^{*}$ (since $P S(J)$ is transitive on pairs $\left.a_{*} \nsim b^{*}\right)$. Let $\theta P \zeta_{a_{*}, c^{*}}=P \zeta_{f_{*}, g^{*}}$ If $f_{*} \nsim e_{1 *}$, we are done by Proposition 6.3. If $f_{*}=e_{1 *}$, we are done by applying Proposition 6.2 to $\theta^{-1}$. (The hypothesis of Proposition 6.2 that there is a point on both $e_{1}^{*}$ and $g^{*}$ is satisfied, since $F^{\prime}$ is a field [6, p. 35].) Thus we can assume that $f_{*} \sim e_{1 *}$ and $f_{*} \neq e_{1 *}$. Let $f=\alpha e_{1}+s[12]+$ $r$ [31], where $n^{\prime}(s)=0=n^{\prime}(r)$ and at least one of $s$ or $r$ is nonzero. By symmetry, assume that $s \neq 0$. Take $t \in \mathfrak{D}^{\prime}$ such that $n^{\prime}(s, t) \neq 0$ and $n^{\prime}(t)=0$.

Consider the nondegenerate quadratic form $Q(x)=T^{\prime}\left(x^{\#}\right)$ on $J_{0}^{\prime}\left(e_{3}\right)$. If $z \in$ $J_{0}^{\prime}\left(e_{3}\right)$ and $Q(z) \neq 0$, let $S_{z} \in \mathfrak{D}\left(J_{0}^{\prime}\left(e_{3}\right)\right)$ be the hyperplane reflection $x \rightarrow x-$ $Q(z)^{-1} Q(x, z) z$. Let $W$ be the orthogonal complement of the span of $e_{1}, e_{2}, s[12]$, and $t$ [12]. Since $n(s)=0$ and $s \neq 0, \mathfrak{D}^{\prime}$ is split and $J_{0}^{\prime}\left(e_{3}\right)$ has Witt index five [8, p. 169]. Thus there is $w \in W$ such that $Q(w)=Q((s+t)[12])^{-1} \cdot S_{w} S_{(s+t)[12]}$ belongs 
to the reduced orthogonal group $\mathcal{D}^{\prime}\left(J_{0}^{\prime}\left(e_{3}\right)\right)$, so it extends to $\eta \in S\left(J^{\prime}\right)$ fixing $e_{3}$ [6, p. 31]. $\eta$ fixes $e_{1}$ and $e_{2}$ and takes $s[12]$ to $-t[12]$. Since $\eta$ fixes each $e_{i}$, it preserves each $\mathfrak{D}^{\prime}[j k]$ [3, Lemma 3.2]. Since $\eta$ preserves $e_{1}$ and $J_{0}^{\prime}\left(e_{1}\right), P \eta$ fixes $e_{1 *}$ and $e_{1}^{*}$ and thus commutes with $P \zeta_{e_{1 *}, e_{i}^{*}}$ [Proposition 2.2]. Applying $\theta^{-1}$ shows that $\theta^{-1} P \eta$ commutes with $P \zeta_{a_{*}, b^{*}}$. Then $\theta^{-1} P \eta$ fixes $a_{*}$, so

$$
P \zeta_{a_{*},\left(\theta^{-1} P \eta\right) c^{*}}=\left(\theta^{-1} P \eta\right) P \zeta_{a_{*}, c^{*}}\left(\theta^{-1} P \eta^{-1}\right) \text {. }
$$

Applying $\theta$ shows that

$$
\theta P \zeta_{a_{*},\left(\theta^{-1} P \eta\right) c^{*}}=P \eta\left(\theta P \zeta_{a_{*}, c^{*}}\right) P \eta^{-1}=P \eta P \zeta_{f_{*}, B^{*}} P \eta^{-1}=P \zeta_{P \eta f_{*}, P \eta g^{*}}
$$

Since $\eta$ preserves each $S^{\prime}[j k], \eta f=\alpha e_{1}-t[12]+u[31]$ for $u \in \mathscr{D}^{\prime}$. The coefficient of $e_{3}$ in $f \times \eta f$ is nonzero, so $f_{*} \nsim P \eta f_{*}$. There is a point on both $c^{*}$ and $\left(\theta^{-1} P \eta\right) c^{*}$, since $F^{\prime}$ is a field [6, p. 35]. We are done by applying Proposition 6.3 with equations (4) and $\theta P \zeta_{a_{*}, c^{*}}=P \zeta_{f_{*}, g^{*}}$

TheOREM 6.5. Let $P H$ be a subgroup of $P \Gamma(J)$ containing $P S(J)$ and let $P H^{\prime}$ be a subgroup of $P \Gamma\left(J^{\prime}\right)$ containing $P S\left(J^{\prime}\right)$. Let $\theta$ be an isomorphism of $P H$ onto $P H^{\prime}$ such that $\theta P S(J)=P S\left(J^{\prime}\right)$. Then there is a collineation or a correlation $P \tau: P J \rightarrow P J^{\prime}$ such that $\theta P \phi=P \tau P \phi P \tau^{-1}$ for all $P \phi \in P H$.

Proof. Let $P S_{m}(J)$ be the kernel of the canonical homomorphism from $P S(J)$ to $P S(J / m J) . P S_{m}(J)$ is the unique largest subgroup of $P S(J)$, and $P S(J) / P S_{m}(J) \cong$ $P S(J / m J)$ [3, Corollaries 6.5 and 7.5]. Thus $\theta$ induces an isomorphism $\theta_{m}$ of $P S(J / m J)$ onto $P S\left(J^{\prime} / m^{\prime} J^{\prime}\right)$. Take $a_{*}, b^{*}, c^{*} \in P J$ such that $a_{*} \nsim b^{*}, a_{*} \nsim c^{*}$, and $b^{*} \nsim c^{*}$. Let $\theta P \zeta_{a_{*}, b^{*}}=P \zeta_{s_{*}, t^{*}}$ and $\theta P \zeta_{a_{*}, c^{*}}=P \zeta_{u_{*}, v^{*}}$ [Theorem 4.4]. If $p: P J \rightarrow$ $P(J / m J)$ and $p^{\prime}: P J^{\prime} \rightarrow P\left(J^{\prime} / m^{\prime} J^{\prime}\right)$ are the canonical maps,

$$
\theta_{m} P \zeta_{p a_{*}, p b^{*}}=P \zeta_{p^{\prime} s_{*}, p^{\prime} t^{*},} \quad \theta_{m} P \zeta_{p a_{*}, p c^{*}}=P \zeta_{p^{\prime} u_{*}, p^{\prime} v^{*}}
$$

Since $p b^{*} \nsim p c^{*}$, Proposition 6.4 implies that either $p^{\prime} s_{*} \nsim p^{\prime} u_{*}$ or $p^{\prime} t^{*} \nsim p^{\prime} v^{*}$, so either $s_{*} \nsim u_{*}$ or $t^{*} \nsim v^{*}$. Thus Propositions 6.2 and 6.3 imply that there is a collineation or a correlation $P \tau: P J \rightarrow P J^{\prime}$ such that $\theta P \zeta_{x_{*} y^{*}}=P \tau P \zeta_{x_{*} y^{*}} P \tau^{-1}$ for all $x_{*} \nsim y^{*}$. If $P \phi \in P H$,

$$
\begin{aligned}
(\theta P \phi) P \tau P \zeta_{x_{*} y^{*}} P \tau^{-1}\left(\theta P \phi^{-1}\right) & =(\theta P \phi) \theta P \zeta_{x_{*} y^{*}}\left(\theta P \phi^{-1}\right)=\theta\left(P \phi P \zeta_{x_{*} y^{*}} P \phi^{-1}\right) \\
& =\theta P \zeta_{P \phi x_{*}, P \phi y^{*}}=P \tau P \zeta_{P \phi x_{*}, P \phi y^{*}} P \tau^{-1} \\
& =P \tau P \phi P \zeta_{x_{*} y^{*}} P \phi^{-1} P \tau^{-1}
\end{aligned}
$$

Proposition 2.2 implies that $(\theta P \phi) P \tau=P \tau P \phi$, as required.

Corollary 6.6. Let $H$ be a subgroup of $\Gamma(J)$ containing $S(J)$ and let $H^{\prime}$ be a subgroup of $\Gamma\left(J^{\prime}\right)$ containing $S\left(J^{\prime}\right)$. Let $\theta$ be an isomorphism of $H$ onto $H^{\prime}$ such that $\theta S(J)=S\left(J^{\prime}\right)$. Then there is a norm semisimilarity $\tau: J \rightarrow J^{\prime}$ and a map $\chi: H \rightarrow$ $R^{\prime}-m^{\prime}$ such that either $\theta \phi=(\chi \phi) \tau \phi \tau^{-1}$ or $\theta \phi=(\chi \phi) \tau \phi^{*-1} \tau^{-1}$ for all $\phi \in H$. If $\phi_{1}, \phi_{2} \in H$,

$$
\chi\left(\phi_{1} \phi_{2}\right)=\left(\chi \phi_{1}\right)\left(\sigma \sigma_{1} \sigma^{-1}\left(\chi \phi_{2}\right)\right)
$$

where $\tau$ is $\sigma$-semilinear and $\phi_{1}$ is $\sigma_{1}$-semilinear. 
Proof. Proposition 2.2 implies that the centralizer of $P S(J)$ in $P \Gamma(J)$ is trivial, so $R-m$ is the centralizer of $S(J)$ in $\Gamma(J)$. Then $\theta$ maps $H \cap(R-m)$ onto $H^{\prime} \cap\left(R^{\prime}-m^{\prime}\right)$ and $\theta$ induces an isomorphism $P \theta$ of $P H$ onto $P H^{\prime}$. By Theorem 6.5, there is a norm semisimilarity $\tau$ of $J$ onto $J^{\prime}$ such that either $P \theta P \phi=P \tau P \phi P \tau^{-1}$ or $P \theta P \phi=P \tau P \phi^{*-1} P \tau^{-1}$ for all $P \phi \in P H$. Thus there is a map $\chi: H \rightarrow R^{\prime}-m^{\prime}$ such that either $\theta \phi=(\chi \phi) \tau \phi \tau^{-1}$ or $\theta \phi=(\chi \phi) \tau \phi^{*-1} \tau^{-1}$ for $\phi \in H$. (5) is equivalent to the condition that $\theta$ is a homomorphism.

Corollary 6.7. (1) The hypothesis that $\theta P S(J)=P S\left(J^{\prime}\right)$ can be deleted from Theorem 6.5 if $P H \subset P G(J)$ and $P H^{\prime} \subset P G\left(J^{\prime}\right)$.

(2) The hypothesis that $\theta S(J)=S\left(J^{\prime}\right)$ can be deleted from Corollary 6.6 if $H \subset G(J)$ and $H^{\prime} \subset G\left(J^{\prime}\right)$. In this case, $\chi$ is a homomorphism whose kernel contains $S(J)$.

Proof. (1) If $K$ is a group, let $[K, K]$ denote the subgroup generated by the commutators of elements of $K .[S(J), S(J)]=S(J)$ [3, equation (v) and Corollary 6.5]. It is immediate that $[G(J), G(J)] \subset S(J)$, so $[P H, P H]=P S(J)$ and $\left[P H^{\prime}, P H^{\prime}\right]=P S\left(J^{\prime}\right)$. Hence $\theta P S(J)=P S\left(J^{\prime}\right)$. (2) As above, $[H, H]=S(J)$ and $\left[H^{\prime}, H^{\prime}\right]=S\left(J^{\prime}\right)$, so $\theta S(J)=S\left(J^{\prime}\right)$. (5) and the assumption that $H \subset G(J)$ imply that $\chi$ is a homomorphism. $\chi(S(J))=1$, since $S(J)=[H, H]$ and $R^{\prime}-m^{\prime}$ is abelian.

COROLlaRY 6.8. (1) The hypothesis that $\theta P S(J)=P S\left(J^{\prime}\right)$ can be deleted from Theorem 6.5 if $R=F$ and $R^{\prime}=F^{\prime}$ are fields.

(2) The hypothesis that $\theta S(J)=S\left(J^{\prime}\right)$ can be deleted from Corollary 6.6 if $R=F$ and $R^{\prime}=F^{\prime}$ are fields.

Proof. (1) Since $P S(J)$ is a normal subgroup of $P \Gamma(J)$ and $\theta P H=P H^{\prime} \supset$ $P S\left(J^{\prime}\right), \theta P S(J)$ is normalized by $P S\left(J^{\prime}\right)$. Since $F^{\prime}$ is a field, any proper subgroup of $P \Gamma\left(J^{\prime}\right)$ normalized by $P S\left(J^{\prime}\right)$ contains $P S\left(J^{\prime}\right)$ [3, Corollary 7.2]. Thus $\theta P S(J)$ contains $P S\left(J^{\prime}\right)$, and replacing $\theta$ by $\theta^{-1}$ gives the reverse containment. (2) Since $S(J)$ is a normal subgroup of $\Gamma(J)$ and $\theta H=H^{\prime} \supset S\left(J^{\prime}\right), \theta S(J)$ is normalized by $S\left(J^{\prime}\right)$. Since $F^{\prime}$ is a field, any subgroup of $\Gamma\left(J^{\prime}\right)$ normalized by $S\left(J^{\prime}\right)$ is either contained in $F^{\prime}-0$ or contains $S\left(J^{\prime}\right)$ [3, Theorem 7.1]. Since $S(J)$ is nonabelian, $\theta S(J)$ contains $S\left(J^{\prime}\right)$. The reverse containment holds by symmetry.

\section{REFERENCES}

1. E. Artin, Geometric algebra, Wiley-Interscience, New York, 1957.

2. R. Baeza, Quadratic forms over semilocal rings, Lecture Notes in Math., vol. 655, Springer-Verlag, Heidelberg, 1978.

3. R. Bix, Octonion planes over local rings, Trans. Amer. Math. Soc. 261 (1980), 417-438.

4. __ Separable Jordan algebras over commutative rings, Dissertation, Yale Univ., 1977.

5. F. Demeyer and E. Ingraham, Separable algebras over commutative rings, Lecture Notes in Math., vol. 181, Springer-Verlag, Heidelberg, 1971.

6. J. Faulkner, Octonion planes defined by quadratic Jordan algebras, Mem. Amer. Math. Soc. No. 104, (1970).

7. N. Jacobson, Composition algebras and their automorphisms, Rend. Circ. Mat. Palermo, (2) 7 (1958), 55-80. 
8. __ Structure and representations of Jordan algebras, Amer. Math. Soc. Colloq. Publ., vol. 39, Amer. Math. Soc., Providence, R. I., 1968.

9. K. McCrimmon, The Freudenthal-Springer-Tits construction of exceptional Jordan algebras, Trans. Amer. Math. Soc. 139 (1969), 495-510.

10. T. Suh, On isomorphisms of little projective groups of Cayley planes, Nederl. Akad. Wetensch. Proc. A65 (1962), 320-339.

11. F. D. Veldkamp, Isomorphisms of little and middle projective groups of octave planes, Nederl. Akad. Wetensch. Proc. A67 (1964), 280-289.

Department of Mathematics, The University of Michigan-Flint, Flint, Michigan 48503 